\title{
Cooperative Strategies for Power Saving in Multi-standard Wireless Devices
}

\author{
Firooz B. Saghezchi, Muhammad Alam, \\ Ayman Radwan, and Jonathan Rodriguez \\ Instituto de Telecomunicações \\ Campus Universitário de Santiago, 3810-193 Aveiro, Portugal \\ \{firooz, alam, aradwan, jonathan\}@av.it.pt \\ http://www.av.it.pt/4tell/
}

\begin{abstract}
G}$ is a promising solution for the future mobile Internet through integrating heterogeneous radio access technologies (RATs) based on the Internet Protocol (IP) where multi-standard wireless devices allow mobile users to experience ubiquitous connectivity by roaming across different networks and connecting through the RAT that best suits their traffic requirements. However, holding multiple active interfaces incurs significant power consumption to the wireless devices. This necessitates investigating disruptive techniques for decreasing the power consumption of the $4 \mathrm{G}$ wireless devices. In this paper, we demonstrate how cognitive radio and cooperative communication can be integrated in $4 \mathrm{G}$ networks to conduct wireless devices to either perform vertical handover or execute relaying by exploiting their available short range interfaces (e.g., WiMedia, Bluetooth, etc) to reduce their power consumption while still enabling the required QoS. Simulation and experimental results validate that $4 \mathrm{G}$ wireless devices can double their battery lifetime by adopting the proposed strategies.
\end{abstract}

Keywords: 4G, power saving, multi-standard wireless devices, cognitive radio, cooperative strategies, short range relaying, context-aware.

\section{Introduction}

$4 \mathrm{G}$ is a new paradigm for cellular architecture that not only supports traditional voice service but also promises broadband and ubiquitous Internet for the future. Unlike previous generations of cellular systems that possess a specific air interface and primarily support voice traffic, $4 \mathrm{G}$ is based on Internet Protocol (IP) and aims to bring together the evolving radio access technologies (RATs) to allow mobile users to connect anywhere with better QoS. A 4G user particularly relies on a multi-standard wireless device to stay connected through the heterogeneous radio access network. The device connects through the best interface at any time considering a number of parameters such as the available RATs, user's mobility, traffic type, etc. For instance, if the user wants to make a phone call while travelling with vehicular speed, it can connect through its LTE or WiMax

A. Galis and A. Gavras (Eds.): FIA 2013, LNCS 7858, pp. 284-296, 2013.

(C) The Author(s). This article is published with open access at link.springer.com 
interface. On the other hand, if the user is static or experiences nomadic mobility in an indoor scenario such as a coffee shop or a shopping mall and wants to browse a webpage, it can connect through its WiFi interface.

Energy is becoming a critical resource for the emerging multi-standard wireless devices. Holding multiple active interfaces requires higher power consumption for a wireless device that normally relies on a limited battery for power supply. Furthermore, advanced imaging features (e.g., camera, high-definition display, etc) and GPS/Galileo receivers will increase considerably the power demand of $4 \mathrm{G}$ wireless devices. On the other hand, mobile battery capacity is finite and the progress of battery technology is very slow, with capacity expected to make little improvement in the near future [1]. As a consequence, there exists a continuously growing gap between the energy consumption of the emerging wireless devices and what can be achieved by the battery technology evolution. Indeed, one could imagine that the future wireless devices may lose their freedom to have "true mobile experience" due to becoming restricted in the proximity of power outlets.

As an attempt to reduce the power consumption of the emerging wireless devices, the European Union (EU) has initiated the C2POWER project 2]. This project introduces a novel approach that exploits the advantages achieved by cognitive radio and cooperative communication to prolong the battery lifetime of multi-standard wireless devices. Cognitive radio and cooperative communication have widely been investigated for efficient spectrum usage and improving the wireless link capacity, respectively. However, research and development to apply these techniques for power saving is still at an early stage.

C2POWER assumes that any wireless device is equipped with two radio interfaces: a long range (LR) interface (e.g., LTE, WiMAX, WiFi, etc) and a short range (SR) interface (e.g., WiMedia, Bluetooth, etc). A wireless device always senses the radio environment and communicates with neighbour devices or networks by exchanging context information. Thanks to this context exchange, wireless devices become aware of their environment (e.g., available networks, channel qualities, nearby wireless devices, their battery levels, etc), so they can react appropriately to reduce their power consumption. To this end, a wireless device either performs vertical handover (VHO) to the RAT demanding the least power consumption or joins a nearby cluster and adopts cooperative relaying using a power efficient SR interface. Specifically, in the later case, nearby wireless devices form an ad-hoc network to be embedded with the infrastructure network. This type of network with mixed nature is known as hybrid ad-hoc network (HANET) or multi-hop cellular network (MCN) in the literature.

In the rest of this paper, we address the C2POWER strategies to reduce the power consumption of multi-standard wireless devices highlighting its contributions and achievements. Section 2 illustrates the scenarios; section 3 presents our architecture for context exchange; section 4 discusses power saving strategies and provides a mathematical model based on coalitional game theory; section 5 discusses the simulation results; finally, section 6 concludes the paper. 


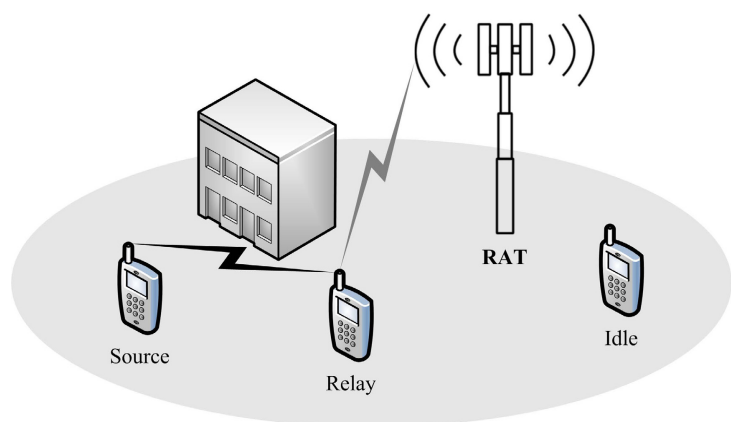

Fig. 1. Scenario 1: A wireless device located in a deep shadowing area relays its traffic through a cooperative SR link. The black link depicts a SR link, while the gray one depicts a LR link.

\section{C2POWER Scenarios}

C2POWER targets three scenarios for power saving. In this section, we illustrate these scenarios.

\subsection{Scenario 1: SR Relaying in Homogeneous RATs}

This scenario addresses a hybrid combination of infrastructure architecture and short range ad-hoc network. As illustrated by Fig. 1, some mobile devices located in the proximity of each other decide to form a cooperative cluster, motivated by power saving strategy or economic incentive. Mobile devices in the same cluster can communicate directly with each other using SR technology. Thanks to the spatial proximity and spatial diversity within a group of cooperative mobiles, there is a high potential for power saving. A particular use case of this scenario is a cooperative strategy where one wireless devices sacrifices part of its energy to instantiate the power saving strategy of the cooperative cluster motivated by a pay-off or other incentives based on a business model.

\subsection{Scenario 2: VHO in Heterogeneous RATs}

This scenario, which is illustrated by Fig. 2, considers that several RATs are available in the location of a multi-standard wireless device. Through this scenario C2POWER investigates strategies and algorithms that assist a system to switch among these interfaces, each with diverse radio characteristics and ranges, to save power. It is pivotal to have efficient handover strategies in place conserving the optimal QoS in addition to reducing the energy consumption. As part of this scenario, C2POWER also considers handover between macro and femtocells. This use case contemplates the specificities of femtocells and handover to/from these cells as well as the improvements that can be achieved by introducing context information (e.g., user location, habits, expected indoor movement, cell coverage, etc). 


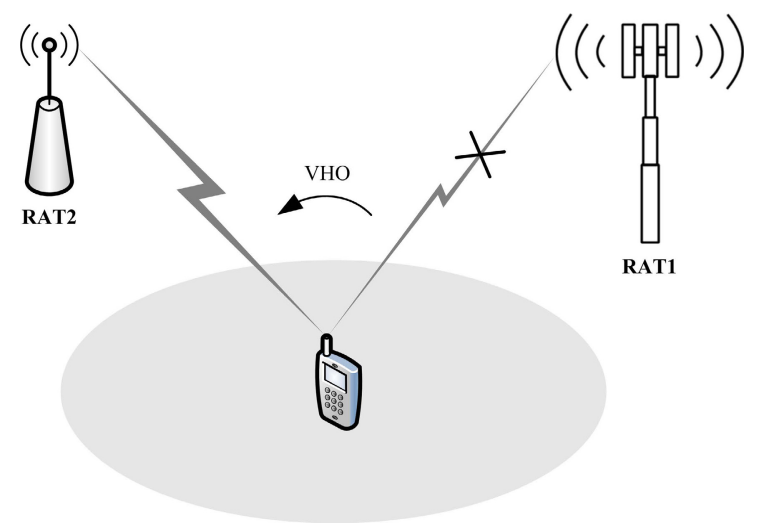

Fig. 2. Scenario 2: A wireless device experiencing bad channel quality to RAT1 performs VHO to RAT2

\subsection{Scenario 3: SR Relaying and VHO in Heterogeneous RATs}

Scenario 3 is a merger of power saving features from Scenario 1 and Scenario 2, allowing cooperation among heterogeneous RATs. As shown by Fig. 3 , the source node is initially connected to RAT1 where it is experiencing bad channel quality and demanding a service (e.g., video streaming) which entails high power consumption when offered by RAT1. Using context-aware capabilities, this node detects a relay node that has access to RAT2 in its SR coverage. This RAT fulfills better the QoS required by the source node and additionally requires less power consumption. Thanks to a low-power SR interface, the source node invites the relay node to establish cooperation. After some negotiation-motivated by a payment or a reputation mechanism-the relay node agrees and a cooperative cluster is established. The relay node then connects to RAT2 and relays to the source node the required traffic over the low-power SR link. The overall power consumption of the cooperative cluster decreases due to this strategy; the amount of power gain depends on the energy efficiency of the cooperative link.

\section{Context-Aware Architecture}

A number of alternative definitions have been applied to the term Context in the literature. For instance, [3] defines context as "any information that can be used to characterize the situation of an entity. An entity is a person, place, or object that is considered relevant to the interaction between the user and the application, including the user and the applications themselves". In [4, contextaware is defined as "location identities of nearby people, objects and changes to those objects".

A number of EU projects have explored context-aware aspects, especially focusing on advantages offered by the context information in wired and wireless communication. Examples are MobiLife [5], SPICE [6], and OPUCE [7, C-CAST 


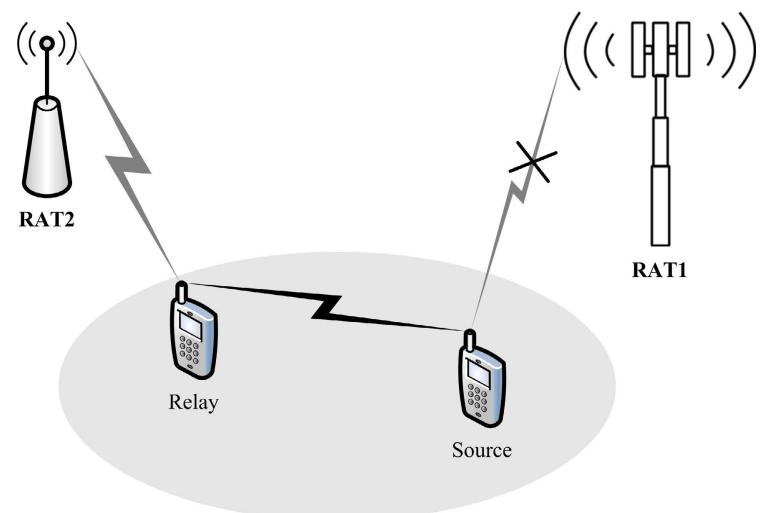

Fig. 3. Scenario 3: A wireless device experiencing bad channel quality to RAT1 performs VHO to RAT2 through a cooperative SR link

8. Integration of context aware systems with mobile multimedia delivery technologies are explored in [9]. A detail of the advantages offered by context and context-aware systems is reported in [10]. In [11, the context is divided and structured into mobile and network context: network context is structured into security, polices, coverage and QoS, while mobile terminal context is related to its capabilities, mobility, application in use, energy and user preferences.

Fig. 4illustrates the proposed context-aware architecture for the context management and utilization in the cognitive engine of the wireless devices. Each device is exposed to the outer world to acquire context information related to other entities (i.e., wireless devices and networks) and feed this information to the context provider. The context provider not only holds the external context but also collects the wireless device's internal context. The context accumulated in the context provider is mostly the raw data which is passed to the context reasoner and filter. The way context provider interacts with the rest of the system depends on whether it is deployed on the network side or on a wireless device. For example, in case of cooperative communication, the context provider receives the data related to nearby wireless devices or available RATs and passes it to the context reasoner. The context reasoner filters, processes, and aggregates the raw data it receives from the context provider and results processed information that is stored in the context repository for future queries. The processed context can also be passed to the context negotiator to exchange with other interested or subscribed wireless devices or networks. Based on publish/subscribe paradigm, the context negotiator gets data via one-time queries to the context repository and to other entities' context negotiators. Thus, context negotiator acts as mediator between two entities (wireless device and network). The processed context information, which is received from the context reasoner, is stored in the database. The context database is then accessed for different purposes via queries. The stored context is useful information to assist wireless devices in decision making while interacting with other entities. The decision engine can access the stored 


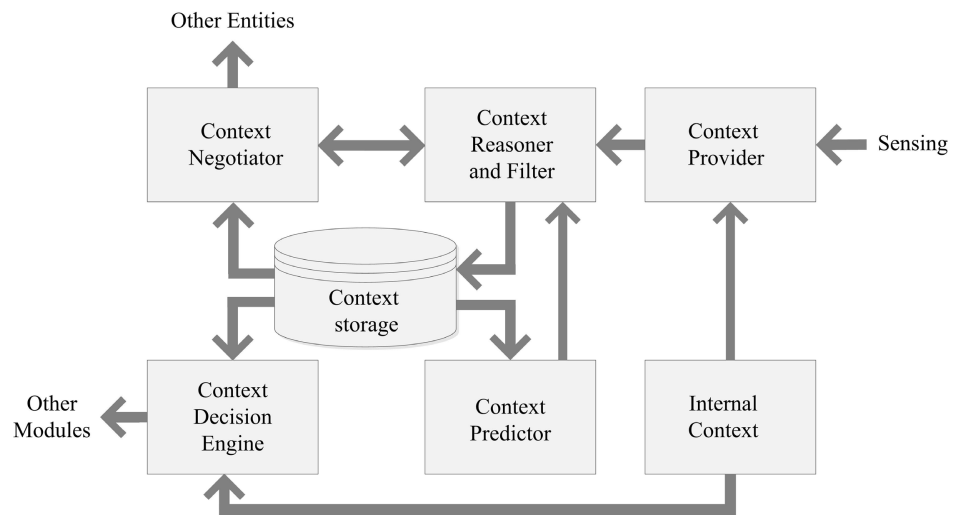

Fig. 4. Context-aware architecture

context and also the internal context to run the decision algorithm. The decisions are based on user preference and the network policies. The outcome of the decision engine can be suggestions for $\mathrm{VHO}$, cooperative SR relaying, etc.

The context predictor provides predictions about future utilization of the context in different cases. Predictions can be made about future battery level for a particular application usage, or it can be related to a wireless device's location after a specific time. The prediction module can be applied to the network side also. For instance, this component can predict location information for disconnected or sleep devices as well as the expected network load in short and medium terms. The context predictor provides this information to the data base through the context reasoner via queries.

\section{Power Saving Strategies}

Utilizing the context information, a cognitive device evaluates the energy cost of all its alternative links to access the network in terms of Joule/Bit and chooses the one that requires the minimum energy while satisfying the required QoS. For a single-hop link, the cost is evaluated by the ratio of the required transmit power to the achieved data rate (i.e., $c=P_{t} / R$ ), and for a two-hop link, it is evaluated as the sum of three terms: the first term is the cost of transmitting through the SR interface for the source; the second term is the cost of receiving through the SR interface for the relay; and the third term is the cost of forwarding through the LR interface for the relay. In the rest of this section, we provide a mathematical model for cooperative SR relaying based on coalitional game theory.

\subsection{Coalitional Game Theory}

A coalitional game in characteristic function form is defined as $\langle N, v\rangle$ where $N$ and $v$ denote the set of players and the characteristic function, respectively $[12$. 
Any subset of $N$ is called a coalition, and the set involving all players is called the grand coalition. The characteristic function assigns any coalition $S$ a real value $v(S)$, called the worth of $S$. Moreover, $v(\emptyset)=0$, where $\emptyset$ denotes the empty set.

Any distribution of the common payoff among the players is called a payoff vector, denoted by $\mathbf{x}=\left(x_{1}, \ldots, x_{n}\right)$. A payoff vector $\mathbf{x}$ is called feasible if it distributes the worth of grand coalition among the players completely; i.e.,

$$
\sum_{i \in N} x_{i}=v(N)
$$

A payoff vector $\mathbf{x}$ is called individually rational if it offers players more payoff than what they can obtain individually; i.e, $x_{i} \geq v(i) \forall i \in N$.

The pre-imputation set is defined as the subset of $\mathbb{R}^{n}$ that contains all feasible payoff vectors; i.e.,

$$
P I(v)=\left\{\mathrm{x} \in \mathbb{R}^{n} \mid \sum_{i \in N} x_{i}=v(N)\right\} .
$$

The imputation set is defined as the subset of $\mathbb{R}^{n}$ that contains all feasible and individually rational payoff vectors; i.e.,

$$
I(v)=\left\{\mathbf{x} \in \mathbb{R}^{n} \mid \sum_{i \in N} x_{i}=v(N) \text { and } x_{i} \geq v(i), \forall i \in N\right\} .
$$

Solution of a coalitional game provides a payoff vector that satisfies the stability of the game. Based on different stability criteria, different solution concepts have been introduced in the literature, namely core, Shapley value, stable set, bargaining set, and kernel. For example, core is defined as the set of payoff vectors that are feasible and cannot be improved upon by any coalition. That is,

$$
c(v)=\left\{\mathbf{x} \in \mathbb{R}^{n} \mid \sum_{i \in N} x_{i}=v(N) \text { and } \sum_{i \in S} x_{i} \geq v(S) \forall S \subset N\right\} .
$$

\subsection{Coalitional Game Model}

For any coalition of wireless devices $S \subset N$, we define the characteristic function as the maximum amount of energy saving that the wireless devices in $S$ can obtain by adopting the cooperative SR relaying strategy. To determine the worth of coalitions, let us partition an arbitrary cluster $C$ into two mutually exclusive sets, namely relays and sources; denoted by $M$ and $N$, respectively. We define a mixed coalition as a subset of $C$ that involves both types of players (relays and sources). For an arbitrary coalition $S \subset N$, if $S$ involves only one player, the worth of coalition is zero since, in such coalitions, the player will find no other player to cooperate with. Generally, if $S \cap M=\emptyset$ or $S \cap N=\emptyset$, the worth of coalition is zero since, in a coalition composed merely of sources (relays), there is no relay (source) to cooperate with and hence there is no opportunity for 
energy saving. Finally, we determine the worth of a mixed coalition by solving an optimization problem with the objective function of maximizing the energy saving of the coalition subject to the resource constraints. For example, for the case of two-hop relaying, this optimization problem can be expressed as follows:

$$
\text { Maximize } v(S)=\sum_{i \in S} \sum_{\substack{j \in S \\ j \neq i}} x_{i j} v(\{i, j\})
$$

Subject to :

$$
\begin{aligned}
& \sum_{j \in N} x_{i j} \leq 1 \forall i=1, \ldots, m \\
& \sum_{i \in M} x_{i j} \leq 1 \forall j=1, \ldots, n \\
& x_{i j} \in\{0,1\} \forall i, j
\end{aligned}
$$

where $x_{i j}$ is a binary decision variable indicating whether relay $i$ should relay source $j$ or not, and $v(\{i, j\})$ is the potential energy saving from cooperation of relay $i$ and source $j$. There are two constraint inequalities: the first one indicates that any relay can relay at most one source, while the second one indicates that any source can either communicate directly or utilize at most one relay to reach the access point. The latter is resulted from the two-hop constraint, which governs that any cooperative link utilizes at most one intermediate relay node.

Once the characteristic function of the game is determined, we can apply one of the solution concepts such as the core solution defined by (4) to solve the game. Solution of the game indeed provides a fair distribution of the common energy saving among the wireless devices within the cooperative cluster so that every player is satisfied-this way the cooperation evolves. Further discussion on this topic is available in [13].

\subsection{Cooperation Enforcement}

In a cooperative cluster, the saved energy is nontransferable. That is, only source nodes enjoy power saving, while relays are incurred some extra power consumption for relaying. Consequently, even if the cooperation can reduce the aggregate power consumption of the cluster, a relay will be reluctant to cooperate unless it is assured that any effort will be compensated. Although reciprocal altruism can be adopted by players to settle this problem, it suffers from lack of trust among players, rendering it highly vulnerable to potential free riding attempts from malicious nodes. Therefore, a mechanism should exist to incentivize cooperative players and avert potential threats of the selfish players; otherwise, the cooperation will collapse. There are two main approaches to address this problem, namely reputation and virtual currency. We apply the latter using a similar approach as in [14] where a virtual central bank (VCB) is adopted to assess the trustworthiness of the players. In this scheme, any wireless device has an energy account in the VCB, with some initial credit, where its efforts are fully recorded 


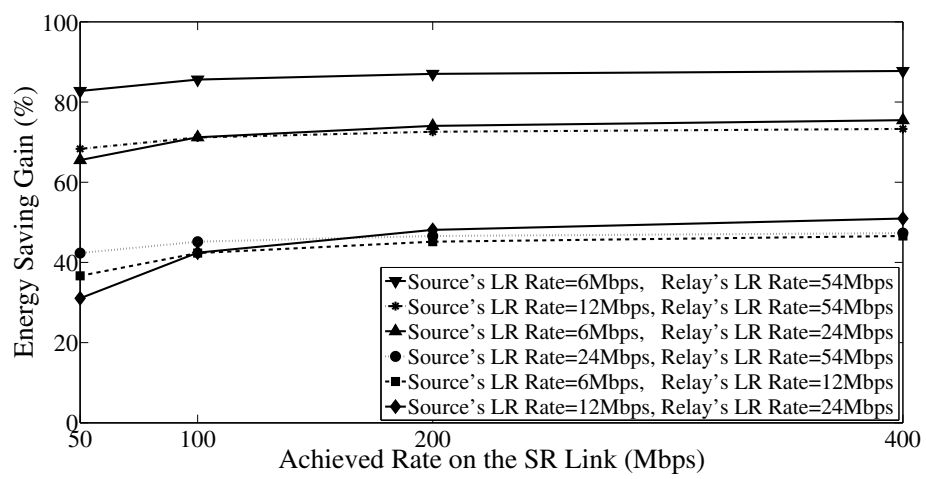

Fig. 5. Energy saving gain from theoretical analysis

and tracked. The VCB rewards cooperative nodes by energy credits, the amount of which is determined by the solution of the game. Moreover, the VCB compensates the relays by rewarding them while charging their corresponding sources by means of energy credit. Receiving a cooperation request from a source device, the VCB checks the device's energy account and entitles it to benefit from others' help if there is enough credit in its account. Finally, to be trustable by all players, the VCB should be implemented in the infrastructure side. Further discussion on this topic is available in [15].

\section{Results and Discussion}

For numerical validation, we focus on Scenario 1 with WiFi and WiMedia interfaces. We define energy saving gain as the ratio of the achieved energy saving from cooperation to the required energy for direct communication. A comprehensive quantitative analysis has been conducted in [16] considering different SR-LR use cases (i.e., WiMedia-WiFi, WiFi-WiMax, and WiFi-WiFi). As a result of this study, Fig. 5 illustrates the achievable energy saving gains for the case of WiFi-WiMedia. As can be seen in this figure, wireless devices can achieve more than $50 \%$ energy saving on average which can even surpass $80 \%$ in some cases when the source node and the relay node possess $6 \mathrm{Mbps}$ and $54 \mathrm{Mbps} \mathrm{LR}$ channels, respectively. Although, this study is a theoretical analysis and overlooks any possibility of establishing the required SR channel between the source and relay nodes, it can shed light on the achievable energy saving limits.

As a first attempt to study the impact of SR and LR channels, a simulation was conducted in [15] for a coffee shop scenario with 5 sources and 5 relays with simplistic Euclidian distance channel models. This study, which considers the WiFi-WiMedia use case, reports an average energy saving gain of $38.8 \%$ with standard deviation $16 \%$ and maximum $76.6 \%$.

To study the energy saving gain in more realistic scenarios, we conducted simulations with stochastic channel models in [17. In this study, for SR channels, 


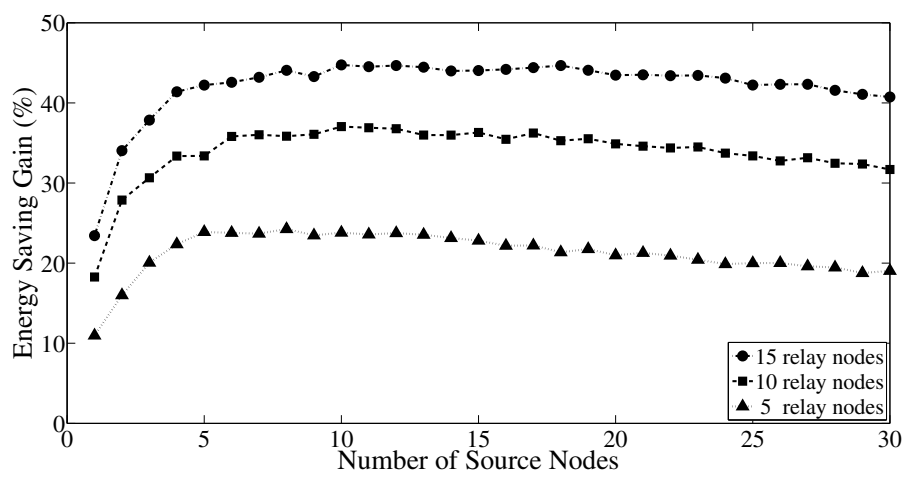

Fig. 6. Energy saving gain for three different numbers of relay nodes when the number of source nodes varies from 1 to 30

we assume line-of-sight (LOS) model with path loss exponent 1.7, while for LR channels, we assume non line-of-sight (NLOS) model with path loss exponent 5, shadowing standard deviation $8 \mathrm{~dB}$, and with flat Rayleigh fading. Fig. 6] illustrates the energy saving gain for three different populations of relays as the number of sources varies between 1 and 30. As can be seen from this figure, for a given number of source nodes, the energy saving gain increases as the population of relays increases. Moreover, when we introduce more source nodes in the simulation area, although the energy saving gain increases in the early stages, it saturates soon after introducing few sources.

To study the impact of relays' density, we vary the total number of nodes from 10 to 100 for three different densities of relay nodes, namely $20 \%, 50 \%$, and $80 \%$., Fig. 7 depicts the result of this simulation. As can be seen from this figure, when the percentage of relays increases from $20 \%$ to $50 \%$, energy saving gain increases significantly; however, the gain improves slightly when the percentage of relays increases from $50 \%$ to $80 \%$. Moreover, the curves are fairly steep until reaching 50 nodes in total, yet their gradients start to decline afterwards. The result indicates that the energy saving gain depends not only on the total population of the nodes but also on the percentage of relays. Furthermore, to avoid intractable relay selection algorithm in terms of running time and context overhead while ensuring a reasonable energy saving gain, appropriate cluster size is estimated in the order of 30-50 nodes. Finally, as an instance, for a cluster of 50 nodes with $50 \%$ relay density, the result indicates that the cooperative SR relaying strategy can reduce the power consumption of the wireless devices by $50 \%$.

For further validation, a demonstrative testbed was designed and implemented within C2POWER project. The demonstrative showcase is dedicated to test SR cooperative strategies for power savings. The testbed consists of C2POWER nodes, which allows the power and energy efficiency to be measured in heterogeneous environment. Each node has two interfaces, namely WiFi and WiMedia. The testbed also includes a WiFi AP. The nodes can connect to the AP either 


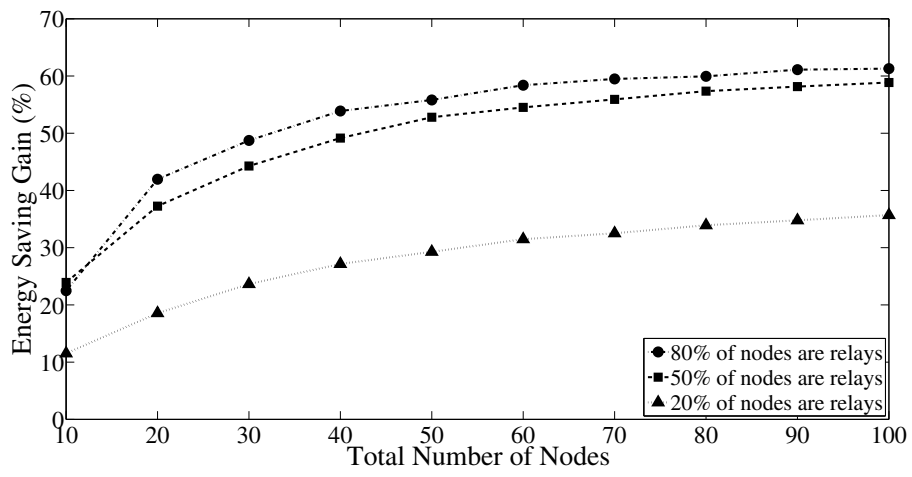

Fig. 7. Energy saving gain for three different densities of relay nodes when the total number of nodes vary from 10 to 100

directly using WiFi connections, or through SR cooperative connections using WiMedia. The testbed was originally designed in a wired environment to provide reliability, stability and reproducibility, but was upgraded to integrate wireless front-end module developed within C2POWER. The testbed has demonstrated good proof of the concept, achieving energy savings, which can reach up to $75 \%$. The testbed is available and was demonstrated at different venues. Due to space limitations, the testbed and its associated results are not fully detailed here.

\section{Conclusion}

The energy required to keep wireless devices connected to the network over extended periods of time dissipates quickly, while the battery technology is not sufficiently mature to anticipate existing and future demands. Without new approaches for energy saving, 4G mobile users will relentlessly be searching for power outlets rather than the network access, and becoming once again bound to a single location. To avoid this problem and to help wireless devices become more environment friendly, this paper addresses strategies to reduce power consumption of multi-standard wireless devices, enabling users to experience true mobile Internet.

We discussed context-aware power saving strategies. These strategies allow the cognitive engine to make the right decision whether or not to initiate cooperative communication based on the foreseen trade off between cooperation cost and potential energy saving. Simulation results validate that wireless devices can reduce their power consumption by $50 \%$ by adopting the proposed strategies. Experimental results through the implemented testbed within C2POWER project also demonstrate the efficiency of the proposed strategies to reduce the power consumption of wireless devices by $75 \%$. 
Acknowledgments. The research leading to these results has received funding from the European Community's Seventh Framework Programme [FP7/20072013] under grant agreement $\mathrm{n}^{\circ} 248577$ [C2POWER] and from the grant of the Fundacão para a Ciência e a Tecnologia (FCT-Portugal), with the reference number: SFRH/BD/79909/2011.

Open Access. This article is distributed under the terms of the Creative Commons Attribution Noncommercial License which permits any noncommercial use, distribution, and reproduction in any medium, provided the original author(s) and source are credited.

\section{References}

1. Anderson, C., Freeman, D., James, I., Johnston, A., Ljung, S.: Mobile Media and Applications, From concept to Cash: Successful Service Creation and Launch. Wiley (2006)

2. C2POWER: ICT-FP7 research project, http://www.ict-c2power.eu/

3. Dey, A.K., Abowd, G.D.: A Conceptual Framework and a Toolkit for Supporting Rapid Prototyping of Context-Aware Applications. J. Human-Computer Interactions 16(2-4), 97-166 (2001)

4. Weiser, M.: The Computer for the 21st Century. Readings in Human-Computer Interaction: Toward the Year 2000, pp. 933-940. Morgan Kaufmann, San Francisco (1995)

5. MobiLife: IST-FP6 Research Project, http://www.ist-mobilife.org

6. SPICE: Service Platform for Innovative Communication Environment, FP6-IST Project, http://www.ist-spice.org

7. OPUCE: Open Platform for User-Centric Service Creation and Execution, FP6-IST Project, http://www.opuce.eu/

8. C-CAST: Context Casting, FP7-ICT Project, http://www.ict-c2power.eu/

9. C-MOBILE: FP6-IST Project, http://c-mobile.ptinovacao.pt/

10. Baldauf, M., Dustdar, S., Rosenberg, F.: A Survey on Context-Aware Systems. International Journal of Ad Hoc And Ubiquitous Computing 2(4), 263-277 (2007)

11. Alam, M., Albano, M., Radwan, A., Rodriguez, J.: Context Parameter Prediction to Prolong Mobile Terminal Battery Life. In: Rodriguez, J., Tafazolli, R., Verikoukis, C. (eds.) Mobimedia 2010. LNICST, vol. 77, pp. 476-489. Springer, Heidelberg (2012)

12. Myerson, R.B.: Game Theory Analysis of Conflict. Harvard University Press (1991)

13. Saghezchi, F.B., Nascimento, A., Albano, M., Radwan, A., Rodriguez, J.: A Novel Relay Selection Game in Cooperative Wireless Networks based on Combinatorial Optimizations. In: Proceedings of IEEE 73rd Vehicular Technology Conference (VTC Spring), Budapest (2011)

14. Zhong, S., Chen, J., Yang, Y.R.: Sprite: A Simple, Cheat-Proof, Credit-Based System for Mobile Ad hoc Networks. In: Proceedings of IEEE INFOCOM 2003, San Francisco (2003) 
15. Saghezchi, F.B., Radwan, A., Nascimento, A., Rodriguez, J.: An Incentive Mechanism based on Coalitional Game for Fair Cooperation of Mobile Users in HANETs. In: Proceedings of IEEE 17th International Workshop on Computer Aided Modeling And Design of Communication Links and Networks (CAMAD), Barcelona (2012)

16. Radwan, A., Rodriguez, J.: Energy Saving in Multi-standard Mobile Terminals Through Short-Range Cooperation. EURASIP Journal on Wireless Communications and Networking, 1-15 (2012)

17. Saghezchi, F.B., Radwan, A., Rodriguez, J.: Energy Efficiency Performance of WiFi/WiMedia Relaying in Hybrid Ad-hoc Networks. Submitted to IEEE 3rd International Conference on Communications and Information Technology (ICCIT), Beirut (2013) 